\title{
NONLINEAR CONVERGENCE ALGORITHM: STRUCTURAL PROPERTIES WITH DOUBLY STOCHASTIC QUADRATIC OPERATORS FOR MULTI-AGENT SYSTEMS
}

\author{
Rawad Abdulghafor, Sherzod Turaev, Akram Zeki, Adamu Abubaker \\ Collage of Information and Communication Technology, International Islamic University \\ Malaysia, 53100, Kuala Lumpur, Malaysia \\ raaac2004@yahoo.com,sherzod@iium.edu.my,akramzeki@iium.edu.my \\ and100adamu@gmail.com
}

Submitted: 26th May 2016; accepted: 21st May 2017

\begin{abstract}
This paper proposes nonlinear operator of extreme doubly stochastic quadratic operator (EDSQO) for convergence algorithm aimed at solving consensus problem (CP) of discrete-time for multi-agent systems (MAS) on n-dimensional simplex. The first part undertakes systematic review of consensus problems. Convergence was generated via extreme doubly stochastic quadratic operators (EDSQOs) in the other part. However, this work was able to formulate convergence algorithms from doubly stochastic matrices, majorization theory, graph theory and stochastic analysis. We develop two algorithms: 1) the nonlinear algorithm of extreme doubly stochastic quadratic operator (NLAEDSQO) to generate all the convergent EDSQOs and 2) the nonlinear convergence algorithm (NLCA) of EDSQOs to investigate the optimal consensus for MAS. Experimental evaluation on convergent of EDSQOs yielded an optimal consensus for MAS. Comparative analysis with the convergence of EDSQOs and DeGroot model were carried out. The comparison was based on the complexity of operators, number of iterations to converge and the time required for convergences. This research proposed algorithm on convergence which is faster than the DeGroot linear model.
\end{abstract}

Keywords: doubly stochastic quadratic operators, nonlinear convergence algorithm, consensus problem, multi-agent systems.

\section{Introduction}

In recent years, a lot of research attention has been directed towards distributed system problems of the group autonomous agents. In such systems, the involved group of agents are expected to operate autonomously and in coordination to reflect the overall systems target goal [1]. These autonomous agents (multi-agent systems) are in fact required to converge to an intended point based on their local interactions. Such convergence can only be achieved through a common agreement between the underlying agents. The agreement in this case, is a consequence of consensus being reached by the respective key players of the group [2]. This issue of reaching consensus and convergence by the respective agents has been a major concern and problem of study by researchers in the field. A particular case of interest is the distributed systems that are comprised of coordinated and controlled multi agents. In this system setup, the agents operate in a large 
scale network and access shared information so as to reach a common agreed upon decision (value) or consensus for an intended common point of convergence.

Consensus problem has many applications is engineering (robots), computer science (distributed computing) and networks (sensors). One of the most challenging problem in the multi-agent system area, is to build a soft protocol that secures all agents to reach a consensus by their negotiation. According to context, the states could be designated as opinions, values, estimates, beliefs, positions, velocities, etc [3].

Consensus problems have a long history in groups, management science, and statistics starting from Parimutuel method for agreement to consensus of individual distributions for subjective probability distribution [4]. Similarly, DeGroot [5] proposed a solution for addressing consensus problems using stochastic matrix and came up with a feasible model called DeGroot Model. This model entails all possibilities by individuals in reaching a certain point of agreement from different opinions. It utilizes transition matrix (stochastic matrix) to prove how consensus is reached. Meanwhile, Berger [6] improved on DeGroots [5] model and proved that reaching a consensus also depends on vector column (initial values) that represents opinions on DeGroots model.

The necessary and sufficient conditions play big role in achieving convergence for consensus related problems. All researches that have studied the convergence of $\mathrm{CP}$ depended on suggested sufficient conditions. The stochastic system also improves the speed of convergence. In [7], the properties of linear convergence of $\mathrm{CP}$ are considered for communication network that is modelled by a random graph scene with Markov based process. The work provided a mathematical proof of necessary and sufficient conditions for achieving an average consensus. The mathematical techniques based on the stability theory of Markov systems, together with the results of matrix and graph theories can be used to test the results of convergence for consensus related problems in a stochastic framework. Moreover, the work in [8] has provided a method for reaching consensus normal nodes, irrespective of the influence of malicious nodes under different assumptions threat, where such derived conditions are applied for robust network based on a novel graphtheoretic approach. The work given in [9] however, has studied the problem of stress consensus for MAS to dynamically change via asymmetric networks with communication delays. On this basis, it was also demonstrated that the original system, finally reached a consensus asymptotically even if communication delays are defined arbitrarily.

A quadratic stochastic operator (QSO), was first Initiated by Bernstein [10] as a nonlinear differential equation, which emerged from some problems of population genetics. The dynamics of some special QSOs on 2D simplex was studied by Vallander [11]. The QSO was later named Volterra, which is the discrete analogue of the nonlinear equation of Lotka-Volterra. The concept and definition of majorization on the other hand was introduced by Olkin and Marshall [12]. Majorization vectors proved as useful tools for the classification of the QSOs and their resulting subclasses. The definition of doubly and dissipative of QSOs are designed through the) majorization theory. The result of Vallander [11] was then extended to the entire finite-dimensional space by Ganikhodzhaev [13] and the dynamics of Volterra QSO were studied successfully in this case as well. However, we cannot say that all QSOs are of Volterra-type, and the non-Volterra dynamics of QSO are still open. Basic properties of majorization and doubly stochastic matrices have been studied by Ando [14]. The limit behaviour of trajectories of QSOs was completely studied on 1D simplex by Lyubich et al. [15], where it was shown that the limit of any initial values is a finite set. The related problem in higher dimensions even on 2D simplex is still an open complexity problem. More general definition and improvement of the necessary and sufficient condition of EDSQOs has been obtained by Ganikhodzhaev [16] under the name of bistochastic operators. A described overview of the results and several open problems of the theory of QSOs were discussed in the work of Ganikhodzhaev and Rozikov [17]. The concept of the class of EDSQOs was introduced by Ganikhodzhaev and Shahidi [18] and the necessary and sufficient conditions for EDSQOs were studied in the same work.

It has been shown that there are 37 operators up to permutation of components of EDSQOs on 2D simplex [19]. The class of extreme doubly 
stochastic quadratic operators is quite large, and consequently the study of the limit behaviour becomes complicated. Shahidi [20] introduced the notions of dissipative quadratic stochastic operator. QSOs along with their respective sufficient conditions. Shahidi [21] also proved that the number of extreme points of the set of EDSQOs is 37. It was obtained that up to permutation matrices; there are 222 extreme points $[19,22]$. The concept of EDSQOs based on majorization theory was then introduced in [23] including the proof on their related necessary conditions. Further, the notion of dissipative quadratic stochastic operators on infinite-dimensional simplex was defined in [24], where the limit behavior of the trajectories and the fixed points of dissipative QSOs were studied with the results obtained on the conditions of convergence. Moreover, the classification of the limit behavior of trajectories of dissipative QSOs on a finite-dimensional simplex is fully studied [25, 26]. It was proved that any dissipative QSO has either unique or infinitely many fixed points. The limit behavior of the trajectories of some extreme EDSQOs on 2D simplex was discussed [27]. It was proved that the trajectories of some extreme EDSQOs tend to the center on the 2D simplex. Further, a Lyapunov based example was provided for the EDSQOs on a finite dimensional simplex. In fact, the control of MAS has accepted the theory of DSQOs [28, 29].

The nonlinear model of EDSQOs is subclass of QSOs, where the EDSQOs are built based on the QSOs and majorization theories. Furthermore, it has been proved that the QSOs investigated are characterized by fast convergence for $\mathrm{CP}$ in multiagent systems by using sufficient conditions of doubly stochastic matrix and distributed matrices. Unfortunately, the functions cost of the QSOs involves very complicated calculations and takes much time to exit after convergence. This is due to the fact that the function for each agent is polynomial.

Therefore, in our paper we propose a model of EDSQOs and compare it with the oldest linear model of DeGroot for CP that was given in [5].

\section{The Consensus Problem in MAS}

A multi-agent system in the most general sense, is defined as a network system of several au- tonomous agents that are capable of local interactions [30]. Each agent is assumed to hold a state regarding a certain quantity of interest. According to the context, states of agents could be opinions, values, beliefs, positions, speed, etc [3]. The agents update their states based on the algorithm by using protocols which could be linear or nonlinear with local interaction among agents and their respective neighbors $[31,2]$. In general, the meaning of consensus is a convergence of all states of agents to a certain value in time of all updated statuses in MAS algorithms [32]. The distributed algorithm for the calculation of averages related to convergence is of high significance and has been well studied in related works. These algorithms require updates of each agents status as a convex combination of the present states of their neighbors and their own [2]. The application of consensus comes up in many areas of research; in biology, the dynamic of consensus is studied for the behavior of flocking of fish and bird schools, etc. [33], where consensus models can be used to analyze, predict and elucidate the behaviour of flocking. In robotics and control, CP come to light in coordination and cooperation of agents in robots and sensors, where this is an important matter in the application of network environment $[34,35]$. In economics, the consensus is used to agree on a common confidence in the price or pricing process. In management science, the $\mathrm{CP}$ has been studied for community of management [5]. In sociology, it is employed for a common language in primal societies and for the dynamics of opinion formation in social networks [36]. In computer science, a distributed computing algorithm is addressed for many typical problems associated with consensus and synchronization where the result of the algorithms is controlled based on the elementary expectations of the distributed formation [37].

\subsection{DeGroot Linear}

A formal system is considered of $n$ agents which are numbered from 1 to $n$. Let state $x_{i}(t)$ scaled agent $i$ at time $t \geq 0$. The algorithm of distributed systems could be either based on continuous or discrete times. A general linear model for distributed discrete time can be given as

$$
x^{(t+1)}=P(t) x(t), t \geq 0,
$$

where $x(t)$ is vector representing the state of agents at time $t$, and $P(t)$ is the chain matrix of the sys- 
tem at time, which is a stochastic matrix where all elements are nonnegative and the sum of each row equals 1. Meanwhile, a general linear model for distributed continuous time can be presented as follows

$$
x^{\prime}(t)=P(t) x(t), t \geq 0,
$$

where $x(t)$ is vector representing the state of agents at time $t$, and $P(t)$ is the chain matrix of the system. The matrix in this case is characterized by the sum of each row being equal to zero and the nondiagonal elements being nonnegative. The meaning of consensus is the convergence of initial values of $x_{i}$ to the same certain value at $t_{i+1}$. In fact, the limit will be different for different agents. In our research, we will focus on the case of a distributed discrete time.

\subsection{QSO Non-linear}

The first study of QSOs can be referred to the work of S.Bernshtein [10]. Over the last 90 years, this theory has evolved and been developed into many classes with significant use to different application areas including physics, biology, and engineering. The theory of QSOs was investigated in [11, 15, 16 and 38]. Vallander [11] established the main result of QSOs on a 2-dimensional simplex. A complete study of the QSOs on onedimensional simplex was by Lyubich [15]. Continuously, Ganikhodzhaev [16] has studied the dynamics of QSOs on infinite-dimensional simplex. Unfortunately, this area has limited applications where all previous studies refer to genetics population. Our proposed approaches in this research is to apply this nonlinear model CP in MAS. This model is a nonlinear which considers a set of points $x=\left(x_{1}, x_{2}, \ldots, x_{m}\right), \sum_{i=1}^{m} x_{i}=1$ and $x_{i} \geq 0$ for $i=$ $1,2, \ldots, m$. We define the $(m-1)-$ dimensional simplex as follows

$$
\begin{array}{r}
S^{m-1}=\left\{x=\left(x_{1}, x_{2}, \ldots, x_{m}\right) \in R^{m}: x_{i} \geq 0,\right. \\
\left.\forall i=1,2, \ldots, m, \sum_{i=1}^{m} x_{i}=1\right\} .
\end{array}
$$

In this case, the general nonlinear model for distributed discrete time is given by:

$$
x^{(t+1)}=P x_{i}^{(t)} x_{j}^{(t)}, t \geq 0
$$

where $x_{i}^{(t)}$ and $x_{j}^{(t)}$ is the row and column of points respectively and $P=\left(P_{1}\left|P_{2}\right| \ldots \mid P_{m}\right)$ is a stochastic matrix. Therefore, the nonlinear dynamic system of QSOs for CP in MAS can be written as

$$
\begin{aligned}
V= & \left(\sum_{i, j=1}^{m} P_{i j, 1} x_{i} x_{j}, \sum_{i, j=1}^{m} P_{i j, 2} x_{i} x_{j}, \ldots,\right. \\
& \left.\sum_{i, j=1}^{m} P_{i j, m} x_{i} x_{j}\right) .
\end{aligned}
$$

\section{Proposed Work}

In this Section we provide some preliminaries from majorization theory, and define EDSQOs.The EDSQO is defined on the simplex that was described in Equation (3).

The set int $S^{m-1}=\left\{x \in S^{m-1}: x_{i} \geq 0\right\}$ is called the interior of the simplex. The points $e_{k}=$ $(0,0, \ldots, \underbrace{1}_{k}, \ldots, 0)$ are the vertices of the simplex and the scalar vector $\left(\frac{1}{m}, \frac{1}{m}, \ldots, \frac{1}{m}\right)$ is the center of the simplex.

A quadratic stochastic operator $V: S^{m-1} \longrightarrow S^{m-1}$ is defined as

$$
V(x)=\sum_{i, j=1}^{m} p_{i j, k} x_{i} x_{j}
$$

where $k$ is the number of the transition matrix for each agent $x_{i}$, the $x_{i}$ and $x_{j}$ represent the number of agent in row and column vectors, respectively. The coefficients $p_{i j, k}$ satisfy the following conditions

$$
p_{i j, k}=p_{i j, k} \geq 0, \sum_{k=1}^{m} p_{i j, k}=1, \quad \forall k=1, \ldots, m .
$$

where $k=1,2, \ldots, m$, and the operator can be written in a matrix form as follows

$$
p_{i j, k}=\left(p_{i j, 1}\left|p_{i j, 2}\right| \ldots \mid p_{i j, m}\right),
$$

where the $p_{i j, k}$ is the transition matrix which satisfies the conditions given in the Equation (8). The matrices $p_{i j, k}$ are non-negative and symmetric. For any $x=\left(x_{1}, x_{2}, \ldots, x_{m}\right) \in S^{m-1}$, we define $x_{\downarrow}=$ $\left(x_{[1]}, x_{[2]}, \ldots, x_{[m]}\right)$ where $x_{[1]} \geq x_{[2]} \geq \ldots \geq x_{[m]}-$ is a non-increasing rearrangement of $x$. It can be recalled from the works in $[14,12]$ that for two elements $x, y$ of the simplex $S^{m-1}$ the element $x$ is majorized by $y$ and written as $x \prec y$ or $y \succ x$ if the following condition holds

$$
\sum_{i=1}^{m} x_{[i]} \leq \sum_{i=1}^{m} y_{[i]}
$$


for any $k=1,2, \ldots, m-1$. In fact, this definition is referred to as weak majorization [12], the definition of majorization requires that $\sum_{i=1}^{m} x_{[i]}=\sum_{i=1}^{m} y_{[i]}$. However, since we consider points only from the simplex, we may drop this condition.

A matrix $P=\left(p_{i j}\right)_{i, j=1,2, \ldots, m}$ is called stochastic (sometimes bistochastic), if

$$
p_{i j} \geqslant 0, \sum_{i=1}^{m} x_{j}=1, \sum_{j=1}^{m} x_{i}=1, \forall i, j=1, \ldots, m .
$$

For a doubly stochastic matrix $P=\left(p_{i j}\right)$, if its entries consist of only 0 's and 1's, then the matrix is a permutation matrix.

A linear map $T: S^{m-1} \longrightarrow S^{m-1}$ is said to be $T$ transform, if $T=(\lambda) I+(1-\lambda) P$ where $I$ is an identity matrix, $P$ is a permutation matrix which is obtained by swapping only two rows of $I$ and $0 \leqslant \lambda \leqslant 1$.

Lemma1. [12] For the concept of majorization and $x, y \in S^{m-1}$, the following assertions are equivalent.

1) $x \prec y$ that is $\sum_{i=1}^{k} x_{[i]} \leq \sum_{i=1}^{k} y_{[i]}, k=1,2, \ldots, m-1$.

2) $x=P y$ for some doubly stochastic matrix $P$.

3) The vector $x$ belongs to the convex hull of all $m$ ! permutation vectors of $y$.

4) The vector $x$ can be obtained by a finite compositions of $T$ - transforms of the vector $y$, that is, there exist $T$ - transforms $T_{1}, T_{2}, \ldots, T_{k}$ such that $x=T_{1} T_{2} T_{3} T_{4} \ldots T_{k}(y)$.

5) The inequality $\varphi(x) \leq \varphi(y)$ holds for any Schurconvex function.

From the above lemma, it follows that doubly stochasticity of a matrix $P$ is equivalent to $P x \prec$ $x \forall x \in S^{m-1}$. Motivated by this in [12], the definition of doubly stochastic operator is then given as follows

Definition: A continuous stochastic operator $V: S^{m-1} \longrightarrow S^{m-1}$ is called doubly stochastic, if $V x \prec x \forall x \in S^{m-1}$. Identity operator, permutation operators (the linear operators with permutation matrix) and $T$ - transforms are all doubly stochastic. Let $V$ be doubly stochastic operator and $x^{0} \in S^{m-1}$. The sequence is infinite $\left\{x^{0}, V\left(x^{0}\right), V^{2}\left(x^{0}\right), \ldots, V^{n}\left(x^{0}\right), \ldots\right\}$ and it is called the trajectory starting at $x^{0}$. Here, $V^{0}\left(x^{0}\right)=x^{0}$ and $V^{n}\left(x^{0}\right)=V\left(V^{n-1}\left(x^{0}\right)\right)$. We denote by $\omega\left(x^{0}\right)$ the set of limit points of the trajectory starting at $x^{0}$ and it is said to be the $\omega$ - limit set of the trajectory starting at $x^{0}$.

The point $x^{0}$ is called p-periodic, if there is a positive integer $\mathrm{p}$ such that $V^{p}\left(x^{0}\right)=x^{0}$ and $V^{i}\left(x^{0}\right) \neq$ $x^{0} \quad \forall i=\overline{1, p-1}$ if $p=1$, then the point is referred to a fixed point.

From the classification of extreme of EDSQOs, we found that all 198 of 222 operators converge to the center for any initial values from the entire simplex. Therefore, we will consider these operators to apply for CP in MAS.

\section{The Consensus Algorithm of ED- SQO}

A fundamental question in $\mathrm{CP}$ is, how the consensus can be guaranteed based on local information exchange with time-varying node interconnections. Therefore, we combine two algorithms to develop a nonlinear reaching convergence algorithm. We start with the design of the NLAEDSQO (Algorithm 1) to define all the convergent EDSQOs. Then we develop the NLCA of EDSQOs (Algorithm 2) to investigate the optimal consensus via nonlinear EDSQOs.

\subsection{The Nonlinear Algorithm of Extreme Doubly Stochastic Quadratic Operator (NLAEDSQO)}

From the theory of QSO [15] and the conditions of EDSQOs in [18] and [23], we define the EDSQOs on 2D simplex by using QSO under the conditions of majorization concept by the NLAEDSQO (see Figure 1) in the following steps: In the first step we define matrices $n$ by $n$, and $n$ is the number of points $\left(P_{1}, P_{2}, \ldots, P_{n}\right)$, where all the elements of the matrices are non-negative Lyubich, real number and symmetric. Moreover, the sum of elements of all three matrices for each row and for each column should be doubly stochastic. The extra necessary conditions are defined for the EDSQOs from the majorization theory, where the diagonal elements of each matrix should be either 1 or 0 while the remaining elements should either be 1 or $\frac{1}{2}$ or $0[18,23]$. Thus the resulting matrices will be $\left(P_{1}, P_{2}, \ldots, P_{n}\right)$. Finally, the sum of entries of any two-by-two sub-matrix should be equal or less than 2 . 
In the next step, we multiply the row vectors $\left(\begin{array}{llll}x & y & \ldots & z\end{array}\right)$ by $\left(P_{1}, P_{2}, \ldots, P_{n}\right)$ matrices to obtain new matrices which are further multiplied by the column vectors of $\left(\begin{array}{c}x \\ y \\ \vdots \\ z\end{array}\right)$, hence EDSQO for the matrices are formed as $(V(x), V(y), \ldots, V(z))$. In turn, we form many operators of EDSQO on infinite dimensional simplex, up to permutations of the matrices where there are 222 [18] [22] extreme points of EDSQOs on 2D simplex. At the end of this procedure, we classify the results to three categories: 198 operators are in center convergent class (CCC), 18 operators are in line convergent class (LCC) and 6 operators are periodic (non-convergent) class (PC). Finally, we take those 198 of the CCC to NLCA to apply them for CCP in MAS.

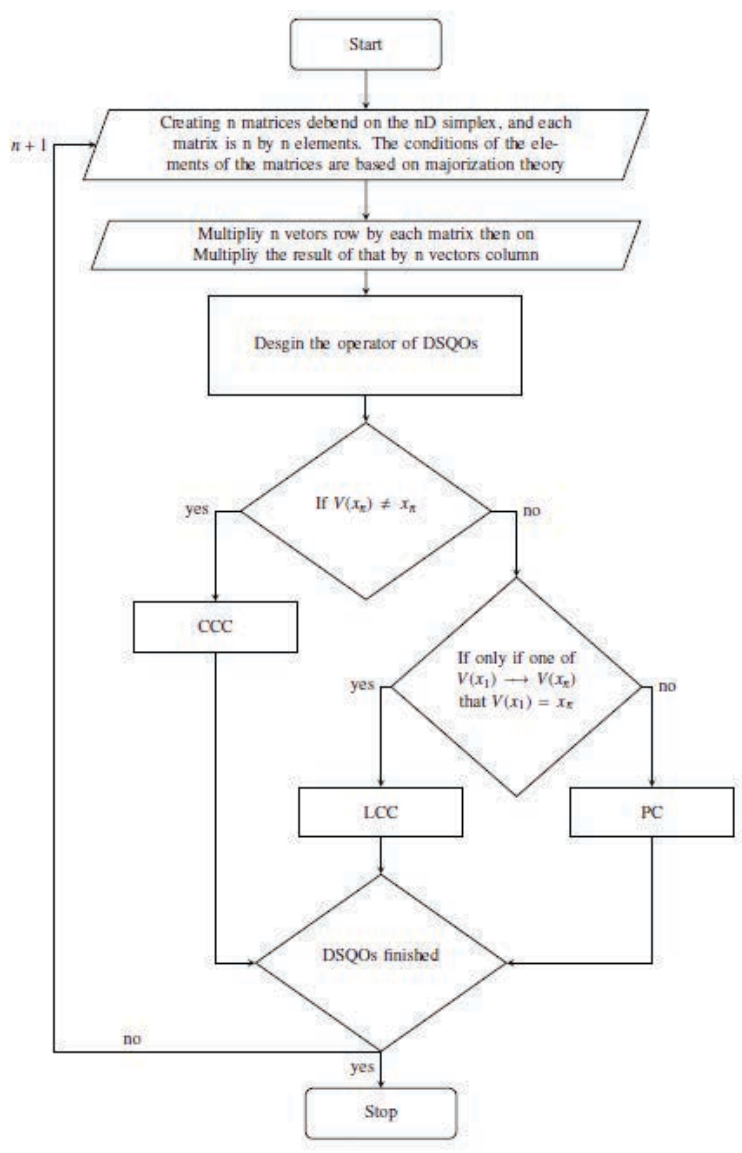

Figure 1. The nonlinear algorithm of extreme doubly stochastic quadratic operator (NLAEDSQO).

\subsection{The Nonlinear Convergence Algorithm (NLCA)}

The derived nonlinear convergence algorithm NLCA of EDSQO operator is as depicted in (see Figure 2) and designed to reflect consensus among agents, where each agent is assigned on appropriate initial values. The aim of the consensus algorithm is to keep the model controlling these values so as to reach a convergence [39].

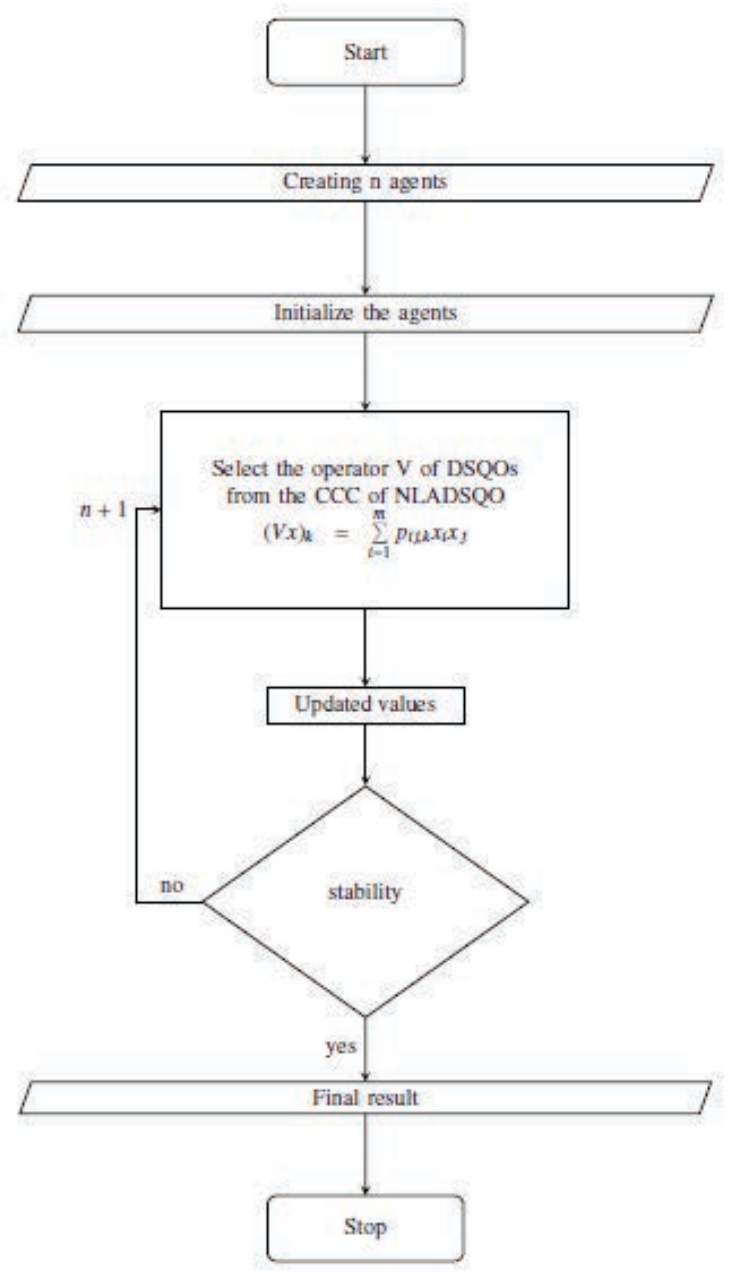

Figure 2. The nonlinear convergence algorithm (NLCA).

Based on the NLCA, we create $n$ agents. Hence, initialize these agents by inserting initial value for each agent. The initial values in this case, are evaluated by using the EDSQO operator. In turn, the state of each agent is subsequently updated as accordingly. the algorithm the checks to see if the states of all agents are stable, then the process would be stopped. However, if the states of agents are not stable, the algorithm then consider the new updated 
state as the initial state and repeats the evaluation (using EDSQO) and updating processes until stability is reached.

In fact, we focus on the NLCA defined by EDSQO to update the states for each variable $x_{0}$ which are of the form: $(V x)_{k}=\sum_{i=1}^{m} p_{i j, k} x_{i} x_{j}$, where $p_{i j, k}$ are doubly stochastic matrices of non-negative real number and symmetric, while $x_{i}$ and $x_{j}$ are the column and row vectors of initial values respectively. In this procedure and at each round $t$, process $(V x)_{k}$ updates $x_{0}$ to some weighted convergence of the values $x_{i}$ it has just received. Each agent has an initial state value and shares this value with their local neighbors in the network. Then the agents have to update their behavior by using local interactions rule. The goal of the update rule is to drive each agent to a state that corresponds to the convergence value of all initial state values. Then we can deduce that the agents have reached convergenceconsensus. More generally, the $\mathrm{CP}$ requires only that the agents converge to a common state value via these local interactions and some rules. Therefore, our NLCA of EDSQO will operate to reflect be agreed upon the common state value that converges to the center of the simplex of all of the agent's initial states.

\section{Discussions and Results}

In this Section, we provide the comparative analysis of the linear DeGroot model, then nonlinear model of QSOs and the nonlinear model of EDSQOs for CP in MAS.

Let us consider the number of agents as three and given by $x, y$ and $z$.

\subsection{DeGroot Linear Model}

We will start with the DeGroot linear model where

$$
\begin{gathered}
x^{(t+1)}=P(t) x(t), \\
x^{(t+1)}=\left(\begin{array}{lll}
a_{11} & a_{12} & a_{13} \\
a_{21} & a_{22} & a_{23} \\
a_{31} & a_{32} & a_{33}
\end{array}\right)\left(\begin{array}{l}
x \\
y \\
z
\end{array}\right),
\end{gathered}
$$

$X=a_{11} x+a_{12} y+a_{13} z$

$Y=a_{21} x+a_{22} y+a_{23} z$

$Z=a_{31} x+a_{32} y+a_{33} z$

with the conditions:
1) $a_{i j} \geq 0$,

2) $a_{11}+a_{12}+a_{13}=a_{21}+a_{22}+a_{23}=a_{31}+a_{32}+$ $a_{33}=1$,

3) $a_{11}+a_{21}+a_{31}=a_{12}+a_{22}+a_{32}=a_{13}+a_{23}+$ $a_{33}=1$.

\subsection{Nonlinear Model of DSQO}

Now, we explore the case of protocols with nonlinear model of DSQO $[26,28]$ that is given by

$$
\begin{gathered}
(V x)=\sum_{i j, k=1}^{m} p_{i j, k} x_{i} x_{j} \\
(V x)=x_{i} p_{i j, 1} x_{j}+x_{i} p_{i j, 2} x_{j}+\ldots+x_{i} p_{i j, m} x_{j},
\end{gathered}
$$

and which will be as follows

$$
\begin{aligned}
& \sum_{i j, k=1}^{m} p_{i j, k} x_{i} x_{j}=
\end{aligned}
$$

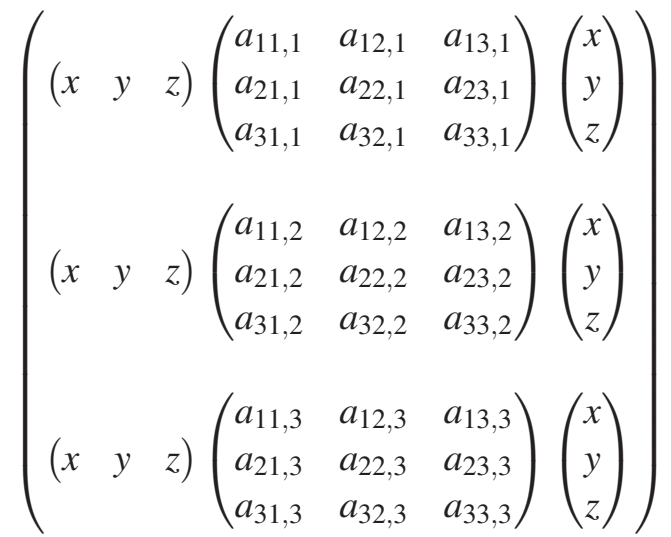

$$
\begin{aligned}
& V(x)=a_{11,1} x^{2}+a_{22,1} y^{2}+a_{33,1} z^{2}+a_{21,1} x y+ \\
& a_{31,1} x z+a_{12,1} x y+a_{32,1} y z+a_{13,1} x z+a_{23,1} y z \\
& V(y)=a_{11,2} x^{2}+a_{22,2} y^{2}+a_{33,2} z^{2}+a_{21,2} x y+ \\
& a_{31,2} x z+a_{12,2} x y+a_{32,2} y z+a_{13,2} x z+a_{23,2} y z \\
& V(z)=a_{11,3} x^{2}+a_{22,3} y^{2}+a_{33,3} z^{2}+a_{21,3} x y+ \\
& a_{31,3} x z+a_{12,3} x y+a_{32,3} y z+a_{13,3} x z+a_{23,3} y z
\end{aligned}
$$

with the same conditions as of the DeGroot model

1) $a_{i j} \geq 0$,

2) $a_{i j, 1}+a_{i j, 2}+a_{i j, 3}=1$, where $i j=i j \forall k$.

\subsection{Nonlinear Model of EDSQO}

Finally, we present the case of protocols of the nonlinear EDSQO model. Formally, the theory of 
the EDSQOs belongs to that of the DSQOs. However, the new sufficient conditions of EDSQOs using majorization theory makes it easier for structure of functions (polynomial) than the DSQO's structure.

$$
\begin{gathered}
(V x)=\sum_{i=1}^{m} p_{i j, k} x_{i} x_{j} \\
(V x)=x_{i} p_{i j, 1} x_{j}+x_{i} p_{i j, 2} x_{j}+\ldots+x_{i} p_{i j, m} x_{j},
\end{gathered}
$$

Remark: According to the dynamic classifications of EDSQOs, we have mentioned that there are three classes of EDSQOs which are CCC, LCC and PC.

We provide some examples for each class of CCC, LCC and PC of EDSQOs on 2D simplex. CCC:

Example of CCC.

The following operator $V: S^{2} \longrightarrow S^{2}$

$$
V_{C C C}=\left\{\begin{array}{l}
V_{C C C}(x)=y z+2 x y \\
V_{C C C}(y)=y z+z^{2}+x^{2} \\
V_{C C C}(z)=2 x z+y^{2}
\end{array}\right.
$$

In the Figure 3, it has been shown that all component vectors $(x, y$ and $z)$ converge to the center. We have found that there are 198 operators of EDSQOs that converge to the center, which are called CCC.

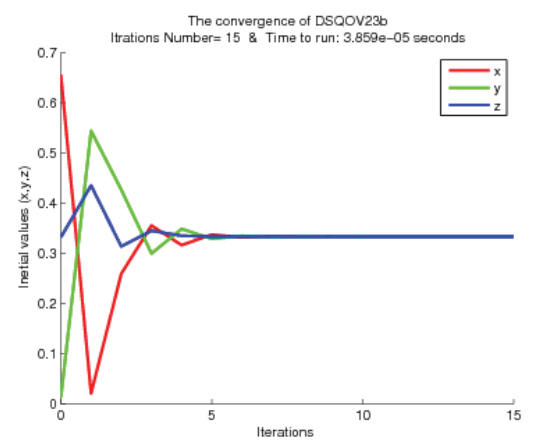

Figure 3. The limit behavior of operator from

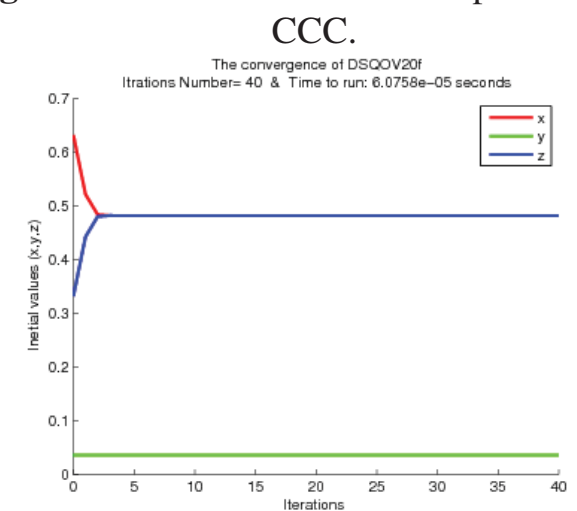

Figure 4. The limit behavior of operator from PC.

\section{Example of LCC.}

The following operator $V: S^{2} \longrightarrow S^{2}$

$$
V_{L C C}=\left\{\begin{array}{l}
V_{L C C}(x)=y z+z^{2}+x^{2} \\
V_{L C C}(y)=x y+y z+y^{2} \\
V_{L C C}(z)=x y+2 y z
\end{array}\right.
$$

In the Figure 5, it can be seen that one of the vector components $y$ is constant, while the other two components $x$ and $z$ converge to the same limit. In this case, we have found 18 operators of EDSQOs, where the limit of one of their components does not change. We call these operators LCC.

\section{Example of PC.}

The following operator $V: S^{2} \longrightarrow S^{2}$

$$
V_{P C}=\left\{\begin{array}{l}
V_{P C}(x)=x z+y z+z^{2} ; \\
V_{P C}(y)=x y+y^{2}+y z ; \\
V_{P C}(z)=x^{2}+x y+x z ;
\end{array}\right.
$$

In the Figure 4, it can be seen that the three vector components never converge. We have found 6 operators under this case, where these operators have a periodic limit. Therefore, it has been called PC.

From the three studies we exclude the classes LCC and PC from applying for CP in MAS. Therefore, we can apply all 198 EDSQOs on 2D of CCC for $\mathrm{CP}$ in MAS.

\section{In the case of the nonlinear model of EDSQO}

$$
\begin{aligned}
& \sum_{i=1}^{m} p_{i j, k} x_{i} x_{j}= \\
& \left(\begin{array}{lll}
\left(\begin{array}{lll}
x & y & z
\end{array}\right)\left(\begin{array}{lll}
a_{11,1} & a_{12,1} & a_{13,1} \\
a_{21,1} & a_{22,1} & a_{23,1} \\
a_{31,1} & a_{32,1} & a_{33,1}
\end{array}\right)\left(\begin{array}{l}
x \\
y \\
z
\end{array}\right) \\
\left(\begin{array}{lll}
x & y & z
\end{array}\right)\left(\begin{array}{lll}
a_{11,2} & a_{12,2} & a_{13,2} \\
a_{21,2} & a_{22,2} & a_{23,2} \\
a_{31,2} & a_{32,2} & a_{33,2}
\end{array}\right)\left(\begin{array}{l}
x \\
y \\
z
\end{array}\right) \\
\left(\begin{array}{llll}
x & y & z
\end{array}\right)\left(\begin{array}{lll}
a_{11,3} & a_{12,3} & a_{13,3} \\
a_{21,3} & a_{22,3} & a_{23,3} \\
a_{31,3} & a_{32,3} & a_{33,3}
\end{array}\right)\left(\begin{array}{l}
x \\
y \\
z
\end{array}\right)
\end{array}\right)
\end{aligned}
$$

The new sufficient conditions of EDSQOs by using majorization theory are:

1) $a_{i j, k} \geq 0$,

2) $a_{i j, 1}+a_{i j, 1}+\ldots+a_{i j, k}=1$, 
3) $a_{i i}=0 \vee 1$,

4) $a_{i j}=0 \vee \frac{1}{2} \vee 1$,

5) $a_{i j}=a_{j i}$,

6) The sum of any sub-block 2 by $2 \leq 2$,

7) The sum of elements of each matrix $=3$.

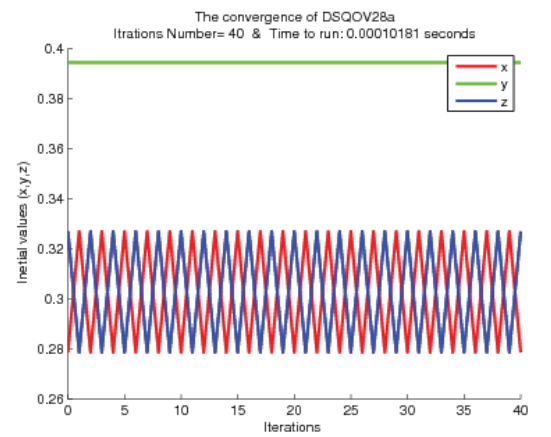

Figure 5. The limit behavior of operator from LCC.

\section{Simulation}

In this case, suppose the initial values of $x, y, z$ are any random values between 0 and 1 , and their summation is equal 1 and given that the transition matrix for DeGroot and DSQO models is a doubly stochastic matrix, while the transition matrix for EDSQO is consisting of 0 or 1 or $\frac{1}{2}$ based on majorization conditions, thus a comparison between DeGroot, DSQO and EDSQO carried out. The results are presented in graphs 9 and 10.
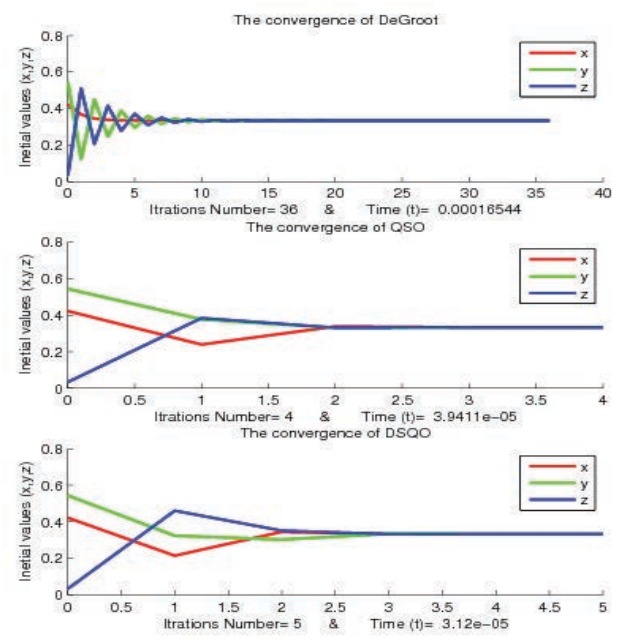

Figure 6. The comparison of convergence between DeGroot linear model, DSQOs nonlinear model and EDSQOs nonlinear model with initial values

$$
(x, y, z) \text {. }
$$

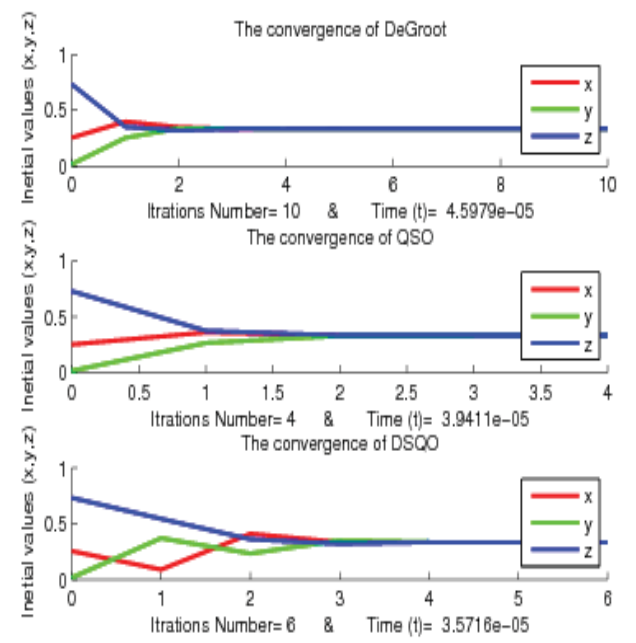

Figure 7. The comparison of convergence between DeGroot linear model, DSQOs nonlinear model and EDSQOs nonlinear model with initial values

$$
(x, y, z) \text {. }
$$

In graphs 9 and 10, it is shown that the convergence time of EDSQOs is faster than that of DeGroot and DSQOs.

The disadvantages of the linear DeGroot model:

1. The linear consensus cannot always be achieved because of the fact that many physical systems in engineering have a special type of consensus problem $[40,41]$.

2. The linear protocol of DeGroot model needs more iterations resulting in more time to converge.

The disadvantages of the nonlinear DSQO model: Since every agent member of the group has a positive subjective distribution, it often makes the calculation highly complex, because each agents has $m^{2}$ product to run in each iteration.

The advantages of the proposed nonlinear protocol based on EDSQO:

1. EDSQO is a nonlinear model; therefore, it is a more accurate model for many physical systems.

2. It requires a few iterations to converge.

3. The transition matrix for each agent does not necessarily need to be stochastic or doubly stochastic as restricted conditions. 
4. The operators are very easy to calculate because the values of the transition matrices are $0,1 / 2$ or 1 , which make all the coefficients equal to one to update the status for each agent, and each agent has only $m$ product to run in each iteration.

\subsection{The Simulation of The Convergence Consensus of Some EDSQOs}

We show some examples of EDSQOs in the following [18, 42]

$$
\begin{aligned}
& V_{1}=\left\{\begin{array}{l}
V_{1}(x)=x^{2}+y^{2}+x z ; \\
V_{1}(y)=z^{2}+x y+x z ; \\
V_{1}(z)=x y+2 y z ;
\end{array}\right. \\
& V_{2}=\left\{\begin{array}{l}
V_{2}(x)=x^{2}+y^{2}+y z ; \\
V_{2}(y)=z^{2}+x z+y z ; \\
V_{2}(z)=x z+2 x y ;
\end{array}\right. \\
& V_{3}=\left\{\begin{array}{l}
V_{3}(x)=y^{2}+z^{2}+x z ; \\
V_{3}(y)=x^{2}+x y+x z ; \\
V_{3}(z)=x y+2 y z ;
\end{array}\right. \\
& V_{4}=\left\{\begin{array}{l}
V_{4}(x)=y^{2}+z^{2}+x y ; \\
V_{4}(y)=x z+y z+y z ; \\
V_{4}(z)=x^{2}+x y+x z ;
\end{array}\right. \\
& V_{5}=\left\{\begin{array}{l}
V_{5}(x)=y^{2}+x z+y z ; \\
V_{5}(y)=x^{2}+x y+y z ; \\
V_{5}(z)=z^{2}+x y+x z ; \\
V_{6}=\left\{\begin{array}{l}
V_{6}(x)=y^{2}+x y+x z ; \\
V_{6}(y)=z^{2}+x y+y z ; \\
V_{6}(z)=x^{2}+x z+y z ; \\
V_{7}(y)=x^{2}+x y+y z ; \\
V_{7}(y)=x y+x z+y z ;
\end{array}\right. \\
V_{8}(z)=z^{2}+x y+y z ;
\end{array} ;\right.
\end{aligned}
$$
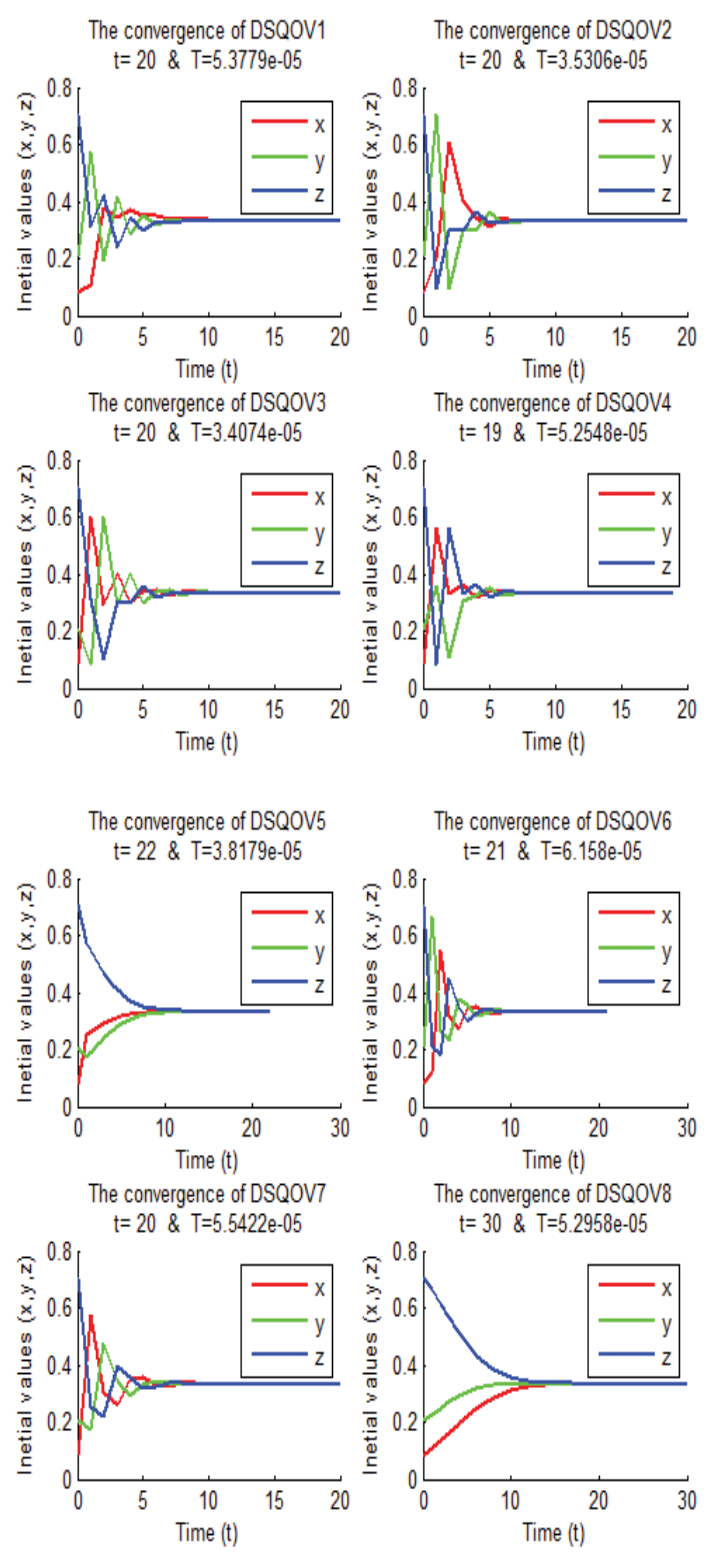

Figure 8. The convergence of the trajectory of EDSQO.

In simulation, we discuss the results of the analysis and then provide general result for all models of DeGroot linear model, DSQOs nonlinear model and EDSQOs nonlinear model (see Figures 6 and 7). At this general result, we observe that the modification of EDSQOs has an advantage over DeGroot linear model and DSQOs nonlinear model. In general, the outcome holds that the nonlinear consensus converges by DSQOs has more complicated function than by EDSQOs. It has been seen that for example the number of agents are three $(x, y, z)$ as well as the number of polynomial is 9 for each agent in the case of DSQOs, while the EDSQO has just 3 
polynomial for each agent. Therefore, the nonlinear EDSQO model has faster time and low complexity calculation than linear DeGroot and nonlinear DSQO models. Finally, we see in Figures 8 and 9 the convergence of some examples of EDSQOs of different initial values. It can be seen clearly that we can design many operators of EDSQO to solve the $\mathrm{CP}$ in MAS. Furthermore, we observe that the convergence time for EDSQOs is still fast in all operators.
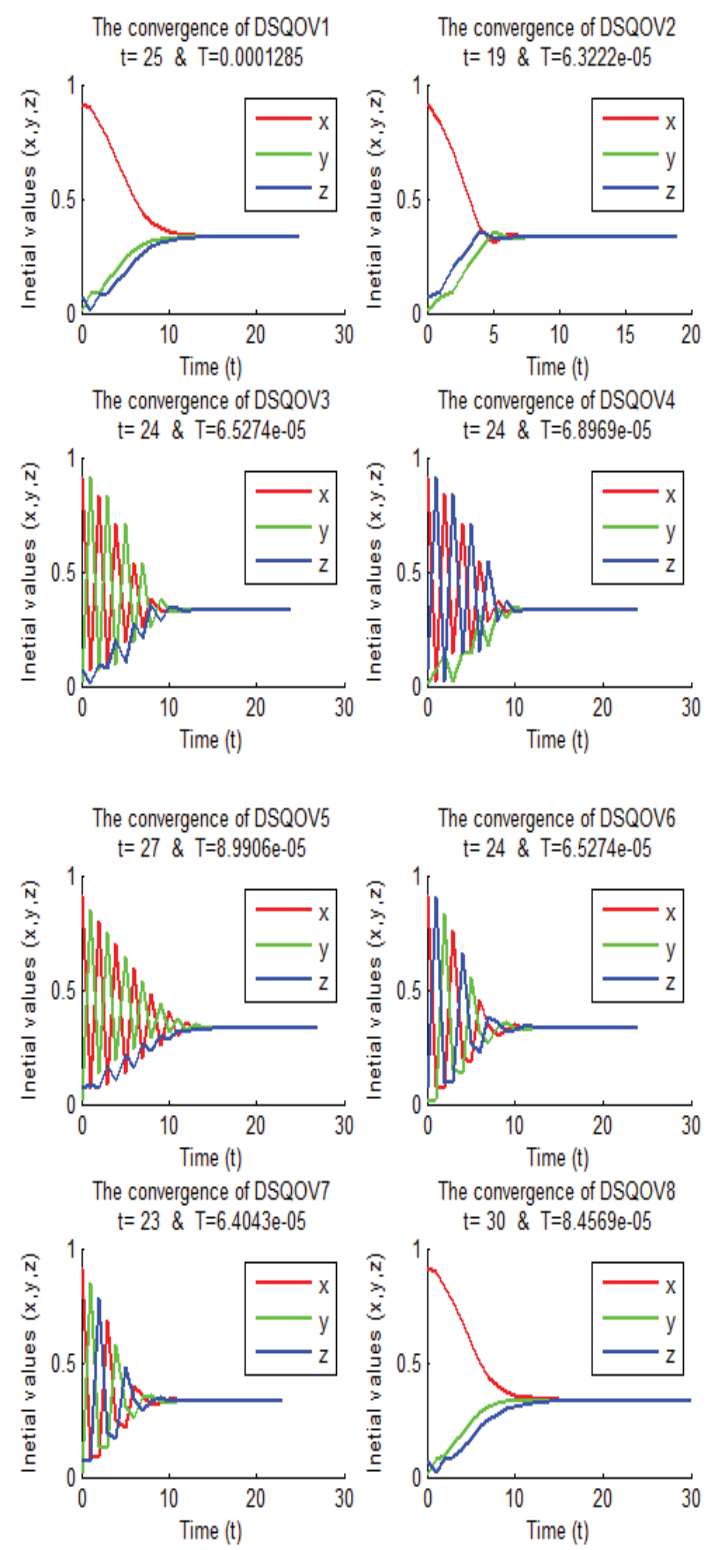

Figure 9. The convergence of the trajectory of EDSQO.

\section{Conclusions}

The novelty of this paper is that a new nonlinear convergence algorithm was presented for a $\mathrm{CP}$ in MAS. The NLCA of EDSQOs is developed based on majorization theory. Generally, we presented the nonlinear extreme doubly stochastic quadratic operator convergence as a modification of QSOs and DeGroot models. We defined two algorithms 1) the NLAEDSQO to design all the convergent EDSQOs and 2) the NLCA of EDSQOs for CP in MAS. Furthermore, we showed the comparison of the convergence NLCA of EDSQOs, DSQOs and DeGroot models for CP. We investigate the NLCA of EDSQOs and found that it is superior than the DeGroot linear and DSQO nonlinear models. Finally, we showed the simulation of NLCA.

\section{ACKNOWLEDGMENT}

We would like to thank Kulliyyah of Information and Communication Technology and the Research Management Center of the International Islamic University Malaysia (IIUM) for their support. This work is supported by the MOHE through IIUM Research Initiative Grant Scheme RIGS16368-0532.

\section{References}

[1] W. Ren, R. W. Beard, and E. M. Atkins, A survey of consensus problems in multi-agent coordination, in American Control Conference, 2005. Proceedings of the 2005, pp. 1859-1864, IEEE, 2005.

[2] Z. Lin, B. Francis, and M. Maggiore, State agreement for continuous-time coupled nonlinear systems, SIAM Journal on Control and Optimization, vol. 46, no. 1, pp. 288-307, 2007.

[3] E. Lovisari and S. Zampieri, Performance metrics in the average consensus problem: a tutorial, Annual Reviews in Control, vol. 36, no. 1, pp. 26-41, 2012.

[4] E. Eisenberg and D. Gale, Consensus of subjective probabilities: The pari-mutuel method, The Annals of Mathematical Statistics, pp. 165-168, 1959.

[5] M. H. DeGroot, Reaching a consensus, Journal of the American Statistical Association, vol. 69, no. 345, pp. 118-121, 1974.

[6] R. L. Berger, A necessary and sufficient condition for reaching a consensus using degroot's method, 
Journal of the American Statistical Association, vol. 76, no. 374, pp. 415-418, 1981.

[7] I. Matei, J. S. Baras, and C. Somarakis, Convergence results for the linear consensus problem under markovian random graphs, SIAM Journal on Control and Optimization, vol. 51, no. 2, pp. 1574-1591, 2013.

[8] H. J. LeBlanc, H. Zhang, S. Sundaram, and X. Koutsoukos, Consensus of multi-agent networks in the presence of adversaries using only local information, in Proceedings of the 1st international conference on High Confidence Networked Systems, pp. 1-10, ACM, 2012.

[9] P. Lin and W. Ren, Constrained consensus in unbalanced networks with communication delays, Automatic Control, IEEE Transactions on, vol. 59, no. 3, pp. 775-781, 2014.

[10] S. Bernstein, Solution of a mathematical problem connected with the theory of heredity, The Annals of Mathematical Statistics, vol. 13, no. 1, pp. 53-61, 1942.

[11] S. Vallander, On the limit behavior of iteration sequence of certain quadratic transformations, in Soviet Math. Doklady, vol. 13, pp. 123-126, 1972.

[12] I. Olkin and A. W. Marshall, Inequalities: Theory of majorization and its applications, Academic, New York, 1979.

[13] R. N. Ganikhodzhaev, On the definition of bistochastic quadratic operators, Russian Mathematical Surveys, vol. 48, no. 4, pp. 244-246, 1993.

[14] T. Ando, Majorization, doubly stochastic matrices, and comparison of eigenvalues, Linear Algebra and Its Applications, vol. 118, pp. 163-248, 1989.

[15] Y. I. Lyubich, D. Vulis, A. Karpov, and E. Akin, Mathematical structures in population genetics, Biomathematics(Berlin), 1992.

[16] R. Ganikhodzhaev, Quadratic stochastic operators, lyapunov functions, and tournaments, Russian Academy of Sciences. Sbornik Mathematics, vol. 76, no. 2, p. 489, 1993.

[17] R. Ganikhodzhaev and U. Rozikov, Quadratic stochastic operators: Results and open problems, arXiv preprint arXiv:0902.4207, 2009.

[18] R. Ganikhodzhaev and F. Shahidi, Doubly stochastic quadratic operators and birkhoffs problem, Linear Algebra and its Applications, vol. 432, no. 1, pp. 24-35, 2010.

[19] R. Abdulghafor, S. Turaev, A. Abubakar, and A. Zeki, The extreme doubly stochastic quadratic operators on two dimensional simplex, in Advanced
Computer Science Applications and Technologies (ACSAT), 2015 4th International Conference on, pp. 192-197, IEEE, 2015.

[20] F. Shahidi, On dissipative quadratic stochastic operators, Applied Mathematics and Information Sciences, vol. 2, pp. 211-223, 2008.

[21] F. Shahidi, On the extreme points of the set of bistochastic operators, Mathematical Notes, vol. 84, no. 3, pp. 442-448, 2008.

[22] R. Abdulghafor, F. Shahidi, A. Zeki, and S. Turaev, Dynamics classifications of extreme doubly stochastic quadratic operators on $2 \mathrm{~d}$ simplex, in Advanced Computer and Communication Engineering Technology, pp. 323-335, Springer, 2016.

[23] F. A. Shahidi, Doubly stochastic operators on a finite-dimensional simplex, Siberian Mathematical Journal, vol. 50, no. 2, pp. 368-372, 2009.

[24] F. Shahidi and M. Abu Osman, The limit behaviour of trajectories of dissipative quadratic stochastic operators on finite-dimensional simplex, Journal of Difference Equations and Applications, vol. 19, no. 3, pp. 357-371, 2013.

[25] F. Shahidi, On infinite-dimensional dissipative quadratic stochastic operators, Advances in Difference Equations, vol. 2013, no. 1, pp. 1-13, 2013.

[26] R. Abdulghafor, F. Shahidi, A. Zeki, and S. Turaev, Dynamics of doubly stochastic quadratic operators on a finite-dimensional simplex, Open Mathematics, vol. 14, no. 1, pp. 509-519, 2016.

[27] F. Shahidi, R. Ganikhodzhaev, and R. Abdulghafor, The dynamics of some extreme doubly stochastic quadratic operators, Middle-East Journal of Scientific Research (Mathematical Applications in Engineering), vol. 13, pp. 59-63, 2013.

[28] R. Abdulghafor, S. Turaev, M. Tamrin, and M. Izzuddin, Nonlinear consensus for multi-agent systems using positive intractions of doubly stochastic quadratic operators, International Journal on Perceptive and Cognitive Computing (IJPCC), vol. 2, no. 1, pp. 19-22, 2016.

[29] R. Abdulghafor, S. Turaev, A. Zeki, and F. Shahidi, The convergence consensus of multi-agent systems controlled via doubly stochastic quadratic operators, in Agents, Multi-Agent Systems and Robotics (ISAMSR), 2015 International Symposium on, pp. 59-64, IEEE, 2015.

[30] L. Panait and S. Luke, Cooperative multi-agent learning: The state of the art, Autonomous Agents and Multi-Agent Systems, vol. 11, no. 3, pp. 387434, 2005. 
[31] A. Ajorlou, A. Momeni, and A. G. Aghdam, Sufficient conditions for the convergence of a class of nonlinear distributed consensus algorithms, Automatica, vol. 47, no. 3, pp. 625-629, 2011.

[32] A. N. Bishop and A. Doucet, Distributed nonlinear consensus in the space of probability measures, arXiv preprint arXiv:1404.0145, 2014.

[33] T. Vicsek, A. Czirók, E. Ben-Jacob, I. Cohen, and O. Shochet, Novel type of phase transition in a system of self-driven particles, Physical review letters, vol. 75 , no. 6 , p. $1226,1995$.

[34] J. N. Tsitsiklis, D. P. Bertsekas, M. Athans, et al., Distributed asynchronous deterministic and stochastic gradient optimization algorithms, IEEE transactions on automatic control, vol. 31, no. 9, pp. 803 812, 1986.

[35] A. Jadbabaie, J. Lin, and A. S. Morse, Coordination of groups of mobile autonomous agents using nearest neighbor rules, Automatic Control, IEEE Transactions on, vol. 48, no. 6, pp. 988-1001, 2003.

[36] F. Cucker, S. Smale, and D.-X. Zhou, Modeling language evolution, Foundations of Computational Mathematics, vol. 4, no. 3, pp. 315-343, 2004.
[37] N. A. Lynch, Distributed algorithms. Morgan Kaufmann, 1996.

[38] S. M. Ulam, A collection of mathematical problems, New York, vol. 29, 1960.

[39] Z.-H. Guan, Y. Wu, and G. Feng, Consensus analysis based on impulsive systems in multiagent networks, Circuits and Systems I: Regular Papers, IEEE Transactions on, vol. 59, no. 1, pp. 170-178, 2012.

[40] G. Cui, S. Xu, F. L. Lewis, B. Zhang, and Q. Ma, Distributed consensus tracking for non-linear multiagent systems with input saturation: a command filtered backstepping approach, IET Control Theory \& Applications, vol. 10, no. 5, pp. 509-516, 2016.

[41] L. Yu-Mei and G. Xin-Ping, Nonlinear consensus protocols for multi-agent systems based on centre manifold reduction, Chinese Physics B, vol. 18, no. 8, p. 3355, 2009.

[42] F. Shahidi, On dissipative quadratic stochastic operators, arXiv preprint arXiv:0708.1813, 2007.

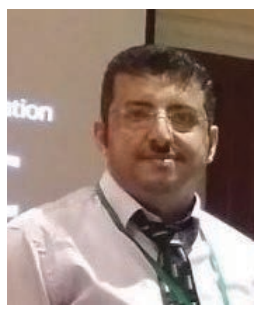

Rawad Abdulghafor received his Bachelor and Master's degrees in Computer Systems Engineering and Informatics from Saint Petersburg Electro-Technical University "LETI", Russia. Now, he is a $\mathrm{PhD}$ candidate in Informatics (Multi-Agent Systems) from the International Islamic University Malaysia, Kuala Lumpur, Malaysia. He has some papers, conferences and chapter book published in the area of stochastic operators and MAS. He has two years undergraduate lecturing experience at Department of Computer Science, Taiz University, Yemen. His research interests are the nonlinear systems for multiple input multiple output, stochastic networks, convergence problem in MAS.

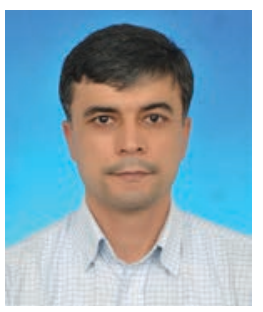

Sherzod Turaev obtained his $\mathrm{PhD}$ in 2010 from Universitat Rovirai Virgili, Tarragona, Spain. He is currently an Assistant Professor at Department of Computer Science, Faculty of Information and Communication Technology, International Islamic University Malaysia. His research interests include Petri net controlled grammars, discretional complexity of formal languages and automata, and weighted DNA computing.

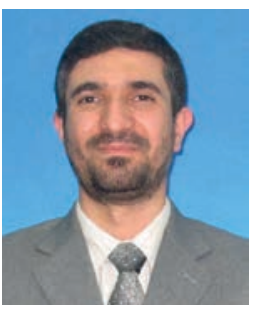

Akram Zeki is an Associate Professor at Kulliyyah of Information and Communication Technology, International Islamic University Malaysia. His research interest including: watermarking, steganography, information security and image processing and multimedia. He is very interested in work in the field of IT and Islam and develop Islamic Applications. He is supervising few master and $\mathrm{PhD}$ students; he is leading few research grants under his university (International Islamic University Malaysia) or under national grant. Dr. Akram published 7 books, 20 articles in journals, 45 book's chapters and more than 75 conference papers. Beside that, he received few awards from university and national level.

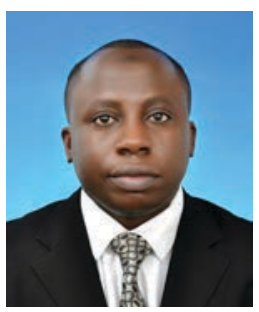

Adamu Abubakar obtained his BSc (Hon) degree in geography, PGD and MSc degree in Computer Science from Bayero University Kano Nigeria. He received his $\mathrm{PhD}$ degree in information and communication technology from International Islamic University Malaysia (IIUM). Presently Dr Adamu is an Assistant Professor at the Department of Information Systems at the International Islamic University Malaysia (IIUM). His research interest is in the areas of 3D mobile map for navigation aid, information retrieval technologies, digital watermarking, steganography, artificial neural network, wireless sensor network and ICT and Islam. 